\title{
Minimal invasive technique in fixation of fracture phalanx by Intramedullary Herbert screw
}

\author{
S.A.Al Traigy, H.E.Farag, W.A.Shawky and H.A.EITaweel \\ Orthopedic, Dept., Faculty of Medicine, Benha Univ., Benha, Egypt \\ E-mail: ElTaweel @gmail.com
}

\begin{abstract}
Hand fractures are very common fractures in orthopedics injuries, and even one of the most common fractures in the upper extremity fractures.there are many different methods of fixation that can be used in the treatment, .Among these methods is fixation of fractures with a Herbert screw, which are suitable for transverse fractures and oblique fractures, which is the subject of this study This study aimed to analyze the clinical and functional results from patients with a diagnosis of unstable reducible extra-articular fracturing of the phalanx with displacement, who underwent surgical treatment by means of a minimally invasive percutaneous osteosynthesis technique using an Herbert screw in order to avoid interfering with the extensor tendon of this finger. Twenty patients, 15 males and 5 females with a mean age of was 36.25 years, (range, 23-57 years). Direct trauma was predominant cause of injury accounting $65 \%$ followed by RTA and trivial trauma $35 \%$. Sixteen patients had transverse fracture $(80 \%)$ and four had oblique fracture $(20 \%)$. the small finger was the most commonly affected digit (12 patients) and other finger (4 middle, 3 index and 1 ring). the mean radiological union time was 7.7 weeks (range 6-10 weeks). Final QuickDASH score was 4.5.The mean TAF was $249^{\circ}$ (range, $\left.245^{\circ}-270^{\circ}\right)$. The mean TAM was $246.25^{\circ}\left(\mathrm{rang}, 235^{\circ}-270^{\circ}\right)$. All the patients follow-up visits were scheduled in the outpatient clinic at 2 weeks, 4 weeks, 6 weeks, 8 weeks, 3 months and 6 months .Herbert screw intramedullary fixation is an effective technique for the treatment of transverse and short oblique proximal and middle phalangeal fractures. We found that it can be applied successfully even in peri-articular fractures of the proximal third of the phalanx, and it a safe method for almost of these fracture types giving a higher ROM and less complication.
\end{abstract}

Keyword: phalanx, Herbert screw, fracture.

\section{Introduction}

Hand fracture is the second most common upper extremity fractures. Phalangeal fractures constitute about $23 \%$ of all fractures occurring from the hand through the forearm [1].

The thumb achieve most of the functions of the hand because of its movement of opposition.The index and the middle finger: help the thumb to achieve the precision grips, The ring finger and the little finger: along with the rest of the hand, are essential for solidly grasping tool-handles on the ulnar side of the hand and thus are vital in strengthening the grip. [2].

The direction of fracture angulation is determined by the imbalance across the fracture site between flexor and extensor forces. A direct blow to the dorsum of the digit will yield a transverse fracture and, depending on the magnitude of the impact, variable degrees of comminution. Combination of bending and axial compression will produce a comminuted fracture with an associated butterfly fragment. A twisting injury will cause a spiral fracture with the fracture line oriented 45 degrees to the shaft of the bone. Combination of torque and axial load will produce a short oblique fracture with variable comminution [3].

Management depends on many factors including the mechanism of injury, fracture pattern, functional requirements of the patient, and surgeon experience [4].

Un-displaced fractures can be treated nonsurgically, but unstable fractures with shortening, angulation, and rotation require surgical intervention, the techniques that have been described vary from provision of relative stability to the principle of absolute stability. A combination of methods is sometimes necessary [5].
Fixation should create a balance between immobilization to prevent fracture displacement and mobilization to prevent stiffness. The main aim of operative fracture fixation is to establish fracture stability and facilitate early mobilization of the hand, to achieve functional recovery as early as possible [6].

There are many methods of fixation such as, open reduction and internal fixation by plate and screw or minimal invasive techniques. Minimally invasive techniques may provide suitable alternatives to plate fixation of phalangeal fractures, thus circumventing their inherent extensive soft tissue dissection. Such options include percutaneous pinning, cerculage wiring, lag screw, or external fixation[7].

Intramedullary headless screws (Herbert Screw) are among the newest options for treatment of unstable phalanx fractures. Positive outcomes following Herbert Screw fixation for metacarpals and phalanges with minimal complications are reported in a small number of recent studies. This procedure is easy technique and present the advantage of not interfering with extensor tendon, so as to avoid tendon adherence and provides immediate stability for early active mobilization [8].

This study aimed to to analyze the clinical and functional results from patients with a diagnosis of unstable reducible extra-articular fracturing of the phalanx with displacement, who underwent surgical treatment by means of a minimally invasive percutaneous osteosynthesis technique using an Herbert screw in order to avoid interfering with the extensor tendon of this finger.. 


\section{Patients and methods}

This study has been conducted at the orthopedic department, faculty of medicine, Benha university hospitals in the period from between December October 2019 to October 2020.

Twenty patients, 15 males and 5 females. With phalangeal fractures were managed by open reduction and internal fixation using intramedullary Herbert screw technique.

\subsection{Inclusion criteria}

To be included in the study, the patient must fulfill all the following criteria:

- Age: > 18 years old.

- Recent fractures: < 3 weeks.

- Transverse, spiral and oblique fracture of shaft.

- Complete radiographic documentation.

- Follow up period for at least 3 months.

\subsection{Exclusion criteria}

The patients with any of the following criteria were excluded from the study:

- Open fractures.

- Vascular injury.

- Tendon injury.

- Compartment syndrome

- Open physeal plate.

- Comminuted fractures

- Articular fractures

- Patients with concomitant hand conditions either traumatic or non- Traumatic (e.g: associated metacarpal or dupuytren's contractures).

\subsection{Preoperative Management and Evaluation} Patient's history

- Personal data: name, age, sex, address, phone number, occupation, hand dominance, medical history and medical habits. .

- Fracture data: mode of trauma, duration from injury, presence of wounds, associated injuries, first aids and any medications received.

\subsection{Clinical examination}

- General examination: the patient was fully examined systematically for any other associated injuries.

- Local examination: complete assessment of the injured hand was done giving attention to open wounds. Documentation of sensory affection by pin prick test and vascularity by capillary refill test was done.

\subsection{Radiographic evaluation:}

- X-rays were include:

- Standard postero-anterior view, lateral view and oblique view of both whole hand \& injured finger.

- Computed tomography (C.T.) :

Whenever seen to be helpful in assessment.

\subsection{Management}

Preoperative measures

- Consent obtained from all patients.

- Preoperative laboratory tests.

- Broad spectrum antibiotic was given preoperatively.

\section{Consent}

Standard consent was taken from the patients

\subsection{Surgical procedure}

Anesthesia

\section{Position}

Patients were anaesthetized by general anaesthesia.

All patients were operated upon while lying in a supine position with the injured upper limb placed on a table position perpendicular to the patient's body.

\section{Operative steps}

A percutaneous approach to the base of the phalanx was used, comprising a $0.5 \mathrm{~cm}$ incision under the lateral face of the extensor tendon in its dorsal region, with a percutaneous minimal approach to the extensor hood affected.

Closed reduction of the fracture of the phalanx was then performed, with the aid of radioscopy.

A guide wire was inserted into the dorsal central part of the base of the phalanx going toward the distal and palmar region of the bone along the whole length with the joint in about $60^{\circ}$ of flexion Fig. (1).
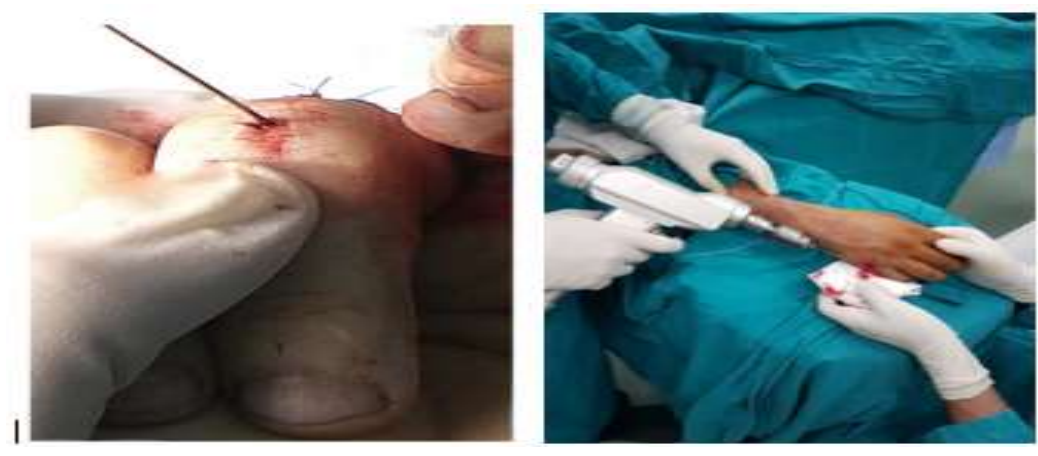

Fig. (1) Insertion of guide wire. 


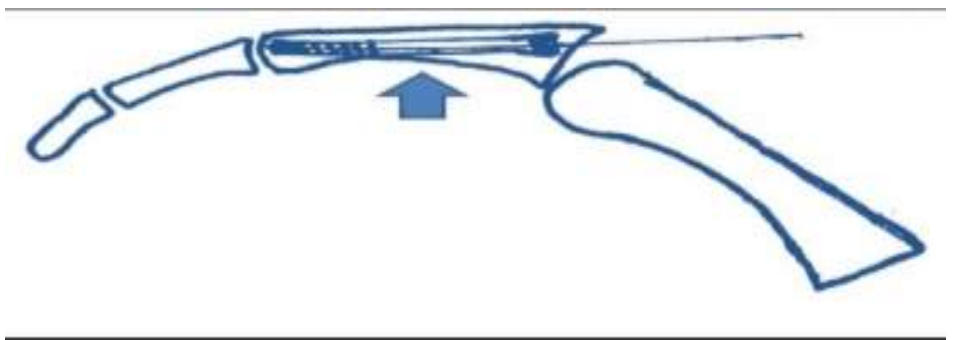

Fig. (2) direction of wire insertion.
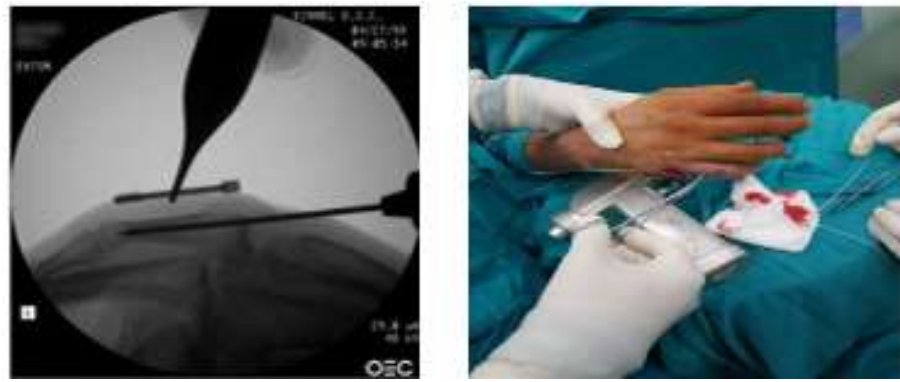

Fig. (3) measure of the size of implant.
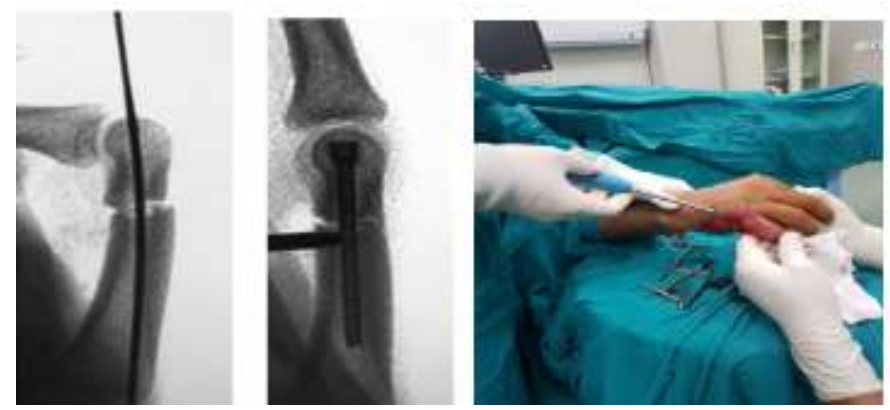

Fig. (4) Herbert screw insertion.

The base of the proximal phalanx was manually gently pushed dorsally to facilitate the positioning of the wire with preservation of the condyles Fig. (2).

After this, the size of the implant was measured and the medullary canal was milled using a specific drill bit Fig. (3).

Once the guide wire was in the correct position and a satisfactory reduction obtained, the Herbert screw was inserted over the guide wire until the screw head reached the metaphysis of the phalanx Fig. (4).

Particular care was taken that the base of the screw did not protrude proximally into the joint and that the tip of the screw reached the distal end of the isthmus of the medullary canal.

\subsection{Postoperative care}

- The Anti-edematous measures: hand elevation, and medications.

- Medications: a broad spectrum oral antibiotic (1 week) and analgesic were given.

- Postoperative x-rays were done for all patients.

- Before discharge, all patients were educated well about the steps and importance of the rehabilitation program and were informed about the dates of follow up visits.

\subsection{Follow up care}

Patient's follow-up visits were scheduled in the outpatient clinic at 2 weeks, 4 weeks, 6 weeks, 8 weeks, 3 months and 6 months for:

\subsection{Clinical evaluation}

- Pain.

- Oedema.

- Stability of the fracture.

- Uprising complications identification and management.

\subsection{Radiological evaluation}

- At 2 weeks, 4 weeks, 6 weeks and 3 months.

- Plain radiographs: postero-anterior, oblique and lateral.

- Delayed union is usually considered at 3-6 months.

- Non-union is usually considered at > 6 months. 


\section{Results}

This study was conducted in orthopedic surgery department, faculty of medicine, Benha university hospitals on twenty patients, 15 males and 5 females. With phalangeal fractures were managed by open reduction and internal fixation using intramedullary Herbert screw technique (Table 1). Direct trauma was predominant cause of injury accounting $65 \%$ followed by RTA and trivial trauma $35 \%$. Sixteen patients had transverse fracture $(80 \%)$ and four had oblique fracture $(20 \%)$. the small finger was the most commonly affected digit (12 patients) and other finger (4 middle, 3 index and 1 ring). the mean radiological union time was 7.7 weeks (range 6-10 weeks).

\section{Regarding time for union}

Regarding time for union (fig-5) the mean radiological union time was 7.7 weeks (range 6-10 weeks). Clinical union preceded or was achieved by the time of radiological union. Ten fractures were united at 6 weeks, six were united in 8 weeks, three in 10 weeks and one in 14 weeks.

All fractures achieved union at time except for one patient with oblique fracture of the proximal phalanx of the Index finger that had surgery 13 days following her injury after patient was initially treated conservatively at another institution and came to our center for a second opinion with fracture displacement.

During the follow-up:- Final quick dash score was (0), Total active motion (TAM) scores were excellent (255) TAM of normal hand (270), (MPJ motion 85, PIPJ motion 90, DIP 80), Return to full duty work after 14 weeks.

Table (1) Demographic characteristics in study population.

\begin{tabular}{lcc}
\hline \multicolumn{3}{c}{ General characteristics } \\
\hline Age (years) & Mean \pm SD & 36.25 \\
Gender & Male $\quad \mathbf{n}(\boldsymbol{\%})$ & $15(75 \%)$ \\
& Female $\quad \mathbf{n}(\boldsymbol{\%})$ & $5(25 \%)$ \\
\hline
\end{tabular}

Post-operative Outcomes Table (1)

Table (2) Post-operative Outcome.

\begin{tabular}{lcccccccccc}
\hline patient & $\begin{array}{c}\text { Return to } \\
\text { full-duty } \\
\text { work } \\
\text { (weeks) }\end{array}$ & $\begin{array}{c}\text { Final } \\
\text { quic } \\
\text { DASH }\end{array}$ & $\begin{array}{c}\text { MPJ } \\
\text { Motion }\end{array}$ & $\begin{array}{c}\text { PIPJ } \\
\text { Motion }\end{array}$ & $\begin{array}{c}\text { Dipj } \\
\text { Motion }\end{array}$ & Taf & $\begin{array}{c}\text { Extension } \\
\text { lag }\end{array}$ & Tam & $\begin{array}{c}\text { Tam o } \\
\text { Normal }\end{array}$ & Out come \\
& & & & & & & & & & \\
$\mathbf{1}$ & 8 & 0 & 85 & 90 & 80 & 255 & 0 & 225 & 260 & excellent \\
$\mathbf{2}$ & 8 & 0 & 90 & 100 & 75 & 270 & 5 & 265 & 270 & excellent \\
$\mathbf{3}$ & 8.5 & 4.5 & 80 & 95 & 75 & 245 & 0 & 245 & 265 & excellent \\
$\mathbf{4}$ & 8 & 2.3 & 90 & 90 & 80 & 255 & 0 & 255 & 260 & excellent \\
$\mathbf{5}$ & 6 & 2.7 & 90 & 100 & 75 & 270 & 0 & 270 & 270 & excellent \\
$\mathbf{6}$ & 10 & 11.4 & 85 & 90 & 80 & 245 & 0 & 245 & 260 & excellent \\
$\mathbf{7}$ & 8 & 3.9 & 90 & 95 & 80 & 265 & 0 & 265 & 270 & excellent \\
$\mathbf{8}$ & 14 & 0 & 85 & 90 & 80 & 255 & 0 & 255 & 270 & excellent \\
$\mathbf{9}$ & 6 & 4.5 & 90 & 85 & 75 & 250 & 0 & 250 & 265 & excellent \\
$\mathbf{1 0}$ & 6 & 2.3 & 80 & 90 & 75 & 245 & 0 & 245 & 257 & excellent \\
$\mathbf{1 1}$ & 6 & 0 & 85 & 85 & 80 & 250 & 0 & 250 & 260 & excellent \\
$\mathbf{1 1}$ & 8 & 13.6 & 80 & 60 & 75 & 215 & 10 & 205 & 260 & excellent \\
$\mathbf{1 2}$ & 6 & 0 & 90 & 90 & 75 & 260 & 5 & 255 & 270 & excellent \\
$\mathbf{1 3}$ & 6 & 0 & 90 & 100 & 75 & 265 & 0 & 265 & 260 & excellent \\
$\mathbf{1 4}$ & 6 & 4 & 90 & 85 & 70 & 245 & 10 & 235 & 260 & excellent \\
$\mathbf{1 4}$ & 6 & 0 & 85 & 80 & 80 & 245 & 0 & 245 & 255 & excellent \\
excellent \\
excellent \\
$\mathbf{1 6}$ & 10 & 2.3 & 90 & 85 & 80 & 255 & 0 & 255 & 270 & \\
$\mathbf{1 7}$ & 10 & 12 & 80 & 60 & 60 & 200 & 20 & 180 & 260 & excellent \\
$\mathbf{1 8}$ & 6 & 11 & 90 & 80 & 55 & 225 & 5 & 220 & 250 & excellent \\
$\mathbf{1 9}$ & 10 & 0 & 90 & 95 & 80 & 265 & 0 & 265 & 270 & \\
$\mathbf{2 0}$ & 7.8 & 3.725 & 86.75 & 86.25 & 75.25 & 249 & 2.75 & 246.25 & & \\
mean & & & & & & & & & &
\end{tabular}




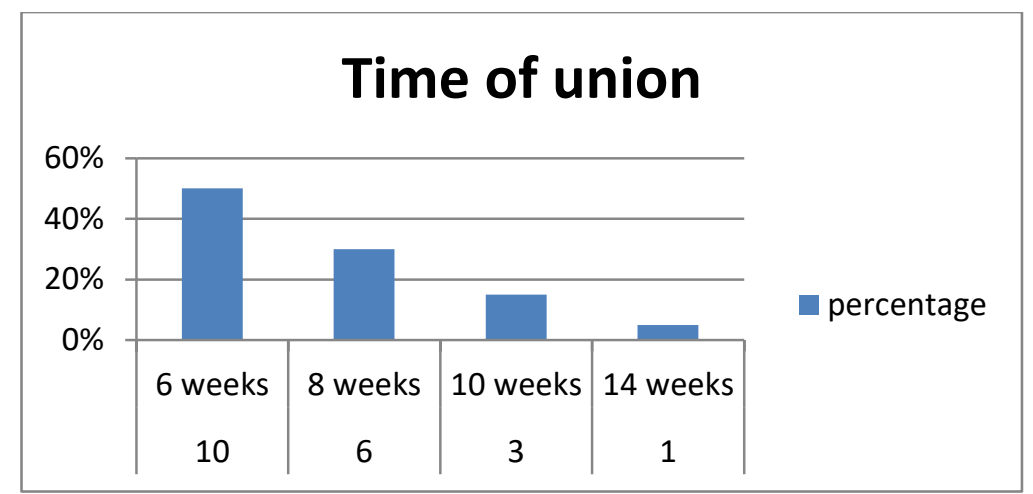

Fig. (5) Time of union.

\section{Regarding of alignment after union}

All fractures united in proper alignment, except one patient who has very proximal fracture to the proximal phalanx of a little finger healed with a dorsal angulation of $15^{\circ}$. The patient did not have any functional problems and did not require further treatment, the patient's hand function was not affected with excellent Total active flexion (TAF) and Total active motion (TAM).

During the follow-up:- Final quick dash score was 4.5, TAM scores were excellent (245) TAM of normal hand (265) MPJ motion 80, PIPJ motion 95, DIP 75), Return to full duty work after 8.5 weeks.

\section{Regarding hand ROM}

At the final evaluation after the fracture union and by a goniometer, TAM and TAF for all involved digits were calculated.

The mean TAF was $249^{\circ}$ (range, $245^{\circ}-270^{\circ}$ ), MP joint motion was $86^{\circ}$ (range, $80^{\circ}-90^{\circ}$ ), and PIP joint motion was $87^{\circ}\left(\right.$ range, $\left.80^{\circ}-100^{\circ}\right)$. and DIP joint was $75^{\circ}$ (range, $60^{\circ}-80^{\circ}$ ). TAF was excellent in 18 digits and was good in 2 digits.

The mean TAM was $246.25^{\circ}$ (rang, $235^{\circ}-270^{\circ}$ ). TAM was excellent in 17 digits, good at 2 digits and one patient has fair TAM. This patient with fair TAM result gave history of a trauma by twisting mechanism from 2 weeks before the surgery. The fracture united in 10 weeks with TAF 180 and fair TAM.

\section{Regarding quick DASH score}

The average disabilities of the arm, shoulder and hand score was 3.72. Complications were observed in 2 fractures, one case complicated with delayed union and union was achieved by 14 weeks, and one case with mal-alignment not affecting the patient hand function.

No clinical mal-unions or rotational deformities were observed. We did not observe any infection or complex regional pain syndrome. We did not observe migration of any screws and no patients required additional intervention.

\section{Discussion}

In this study We present the results of 20 patients with displaced or unstable extra-articular fractures of the base and shaft of the proximal and middle phalanges of the digits of the hand, treated over a period of 3 months with an intramedullary headless compression screw (Herbert screw) and early mobilization with no splinting.

Based on the results of this study, found that the mean TAF was $249^{\circ}$ (range, $245^{\circ}-270^{\circ}$ ), the mean TAM was $246.25^{\circ}$ (rang, $235^{\circ}-270^{\circ}$ ) and the disabilities of the arm, shoulder and hand score (Quick-DASH) was 3.7.

Moreover, and contrary to expectations, this technique achieve a stable fixation that allows early mobilization and avoids stiffness of the MCP and PIP joints. All fractures healed with no major complications .This technique seems to be technically simple, effective and with few drawbacks.

The main drawbacks of this method are that it is suitable only for transverse and short oblique fractures.

This technique was first described by Weiss, for the treatment of phalangeal non-unions[9].

Comparing the results of this study with other studies that were conducted on the same technique, we will find that: A study done by Michael P. Gaspar, Shiv D. Gandhi In (2018) study using Dual Ante-grade Intramedullary Headless Screw Fixation found that total active motion was $258^{\circ}$ (range, $245^{\circ}-270^{\circ}$ ) and QuickDASH score was 3.9 (range,0-13.6). No complications occurred, and no patients required additional intervention [7].

Another study in (2016) done by Giessen etal. Using intramedullary headless screw fixation for fractures of the proximal and middle phalanges in the digits of the hand: The mean TAM of $222^{\circ}$ (range $90^{\circ}-$ $295^{\circ}$ ) was recorded in the fingers. No clinical malunions or rotational deformities were observed. Not observe any infection or complex regional pain syndrome[10].

In (2015) Francisco Del Piñal found that, total active motion was on average $243^{\circ}$ (range, $150^{\circ}$ to $270^{\circ}$ ) for proximal phalangeal fractures. All fractures were healed and within acceptable radiological parameters [11].

Other techniques of fixation of transverse and short oblique fractures of the proximal phalanx include; Plate fixation, K-wires and External fixation. 
Regarding Plate fixation of phalangeal fractures is intended to provide rigid internal fixation to facilitate early movement and there by minimize joint and tendon complications.

However, Kurzen et al, studies found that complications of plate fixation are not rare and they may lead to significant loss of hand function in form of mal-union or non-union and the chief concerns associated with ORPF are tendon adhesions \& loss of motion in the PIP joint [12].

In Brei-Thoma (2015) study retrospectively reviewed the operative and clinical records of 74 consecutive patients having 81 extra-articular basal or diaphyseal fractures of the proximal phalanx, treated with open reduction and plate fixation using minifragment systems, 5 patients suffer from transient symptoms of complex regional pain syndrome (CRPS) like pain combined with trophic changes, vasomotor or sudo-motor dysfunction were noted. There was one patient with a delayed union accompanied by implant failure. Three patients experienced rotational malunion, leading to revision surgery. At final follow-up, or at the time of plate removal and tenolysis, respectively, average TAM of $213^{\circ}$ was found (range $100^{\circ}-285^{\circ}, \mathrm{n}=29$ fractures). Nine fingers were rated to have excellent motion, 9 fingers good motion, and 3 fingers moderate motion. In 8 fingers, however, TAM was still rated as poor. Extension lags of the PIP joints were found in $67 \%$ of all fractured fingers. In 12 patients, plate removal was necessary 4 to 35 months after internal fixation. In seven patients, plate removal and tenolysis were explicitly performed to improve restricted motion[1].

In another study done by Kurzen and et al. in ( 2006), fifty four cases with phalangeal fractures treated with mini-plates and screws, $57 \%$ of the patients developed one or more major complications including; stiffness; twenty two patients $(40.7 \%)$ experienced functionally important stiffness with a ROM $<180^{\circ}$, fracture healing complications in four patients $(6.3 \%)$; delayed union in two patients, and nonunion in two patients, plate loosening in three patients $(5.6 \%)$ or failure in one case $(1.9 \%)$, infection; two patients developed deep infection $(3.7 \%)$, and complex regional pain syndrome in five patients $(9 \%)$. So, they concluded that plate fixation of unstable and complex phalangeal fractures is very efficient and reliable, but not free of potential problems. It demands meticulous handling of soft tissues and does not allow for any technical error [12].

Regarding External fixation; In 2018 Tank and et al, used k-wire and plastic cap of spinal needle cap as a static external fixator device. Clinical results of range of movements and TAM score out of 27 patients $19 \mathrm{had}$ an excellent result (range 220-260), 5 patients had a good result (range 180-219)and 3 with fair result (range 130179) [13].

In another study in (2016) Jafari and et al A study of the management of 52 patients with 56 fractures. (Metacarpal or phalangeal fractures) treated with the
Novel Handmade External Fixator. Clinical results were that none of the cases experienced pin loosening or reduction loss. Of the fractures, $8.9 \%$ malunited due to fracture complexity. The mean dash score was 3.76 . TAM was excellent in $45 \%$ of the fractures; it was good in $7 \%$ and fair in $4 \%$ [14].

In (2006) Margic, found that in a prospective study, used the external fixator in treatment of consecutive patients with closed fractures of the proximal and middle phalanges. Twenty-nine patients had a (TAM > $230^{\circ}$ ). Nine patients were considered to have a poor result. (TAM $<229^{\circ}$ ). Two patients, with grade I and grade IV infections, had poor results. Pressure necrosis of skin in one case caused by Interference of the external fixator with adjacent digits [15].

Regarding Per-cutaneous Kirschner-wires fixation it is the avoidance of an open procedure requiring extensive soft tissue dissection and is very popular because of the short operating time and minimal exposure. Can be performed using trans-articular or peri-articular technique and has disparate results in the literature. Some reports have described good results with bony union and minimal loss of motion.

However, Eberlin (2014) study on management of 43 patients with 50 fractures were identified as having undergone peri-articular percutaneous pinning of proximal phalangeal fractures suggested that , 7 patients had fair results $(17 \%)$, and 1 had a poor result $(2 \%)$, three patients developed lack of flexor pull-through ( $6 \%$ ), two patients developed pin site infections (4\%) [4].

Faruqui (2012) study (Peri-articular and transarticular pinning ), Complications rate are about 32\% with fixed flexion contracture $>15^{\circ}, 20 \%$ with extensor lag and $50 \%$ with flexion loss $>20^{\circ}[16]$.

Singh (2011) ( Percutaneous pinning) study on management of 37 digits reported that Three complications were seen in Group B, which included one each of malunion, digital stiffness and extensor lag. Stiffness rate was about (6\%) [17].

Horton (2003) study found that, the disadvantages of this technique include its often unrecognized technical difficulty, its inability to achieve interfragmentary compression and its potential to tether the soft tissues, especially the lateral bands of the extensor mechanism and infection rate was about $13 \%$ and $20 \%$ with stiffness [18].

\section{Conclusion}

Herbert screw intramedullary fixation is an effective technique for the treatment of transverse and short oblique proximal and middle phalangeal fractures. We found that it can be applied successfully even in peri-articular fractures of the proximal third of the phalanx, and it a safe method for almost of these fracture types giving a higher ROM and less complication. 


\section{References}

[1] P.Brei-Thoma , E.Vögelin ,T. Franz Plate fixation of extra-articular fractures of the proximal phalanx: do new implants cause less problems? Arch Orthop Trauma Surg.vol. 135,pp.439-45,2015.

[2] L.Kapand JI. The Physiology of the Joints Annotated diagrams of the mechanics of the human joints (Volume I). 5th Edition. By Churchill livingstone.vol.8,pp,164-171,1982.

[3] S.Wolfe, R.Hotchkiss ,W.Pederson , S.Kozin , fracture of the metacarpal and phalanges. Green's Operative Hand Surgery, Sixth Edition, Elsevier.vol.2,pp.34-72,2010.

[4] K.Eberlin,A.Babushkina ,J.Neira , C.Mudgal . Outcomes of closed reduction and periarticular pinning of base and shaft fractures of the proximal phalanx. J Hand Surg Am.vol. 39,pp.1524-8, 2014.

[5] C.Anoop, M.Dhamangaonkar,S.Hemant, M.Patankar. Antegrade Joint-Sparing Intramedullary Wiring for Middle Phalanx Shaft Fractures. J Hand Surg Am,2014.

[6]R.Gupta,R.Singh,R.Siwach,S.Sangwan,N.Magu ,R. Diwan . Evaluation of surgical stabilization of metacarpal and phalangeal fractures of hand. Indian J Orthop.vol.41(3),pp.224e229,2007.

[7] P.Michael . Gaspar, D.Shiv , Gandhi, C.Randal, and M.Patrick. Dual Antegrade Intramedullary Headless Screw Fixation for Treatment of Unstable Proximal Phalanx Fractures.AAHS.vol.(1),pp.77-88,2018.

[8] D.Ruchelsman ,S. Puri ,N. Feinberg-Zadek . Clinical outcomes of limited-open retrograde intramedullary headless screw fixation of metacarpal fractures. J Hand Surg Am.vol.39(12),pp.2390-2395,2014.

[9] A.Weiss . Intramedullary Herbert screws for treatment of phalangeal nonunion. Tech Hand Up Extrem Surg.vol. 1,pp.41-7,1997.
[10] T.Giesen,R.Gazzola,A.Poggetti,P.Giovanoli,M Calcagni .Intramedullary headless screw fixation for fractures of the proximal and middle phalanges in the digits of the hand:a review of 31 consecutive fractures. J Hand Surg Eur,2016.

[11] F.Del Piñal ,E.Moraleda ,G.Ruas JS, de Piero ,L.Cerezal. Minimally invasive fixation of fractures of the phalanges and metacarpals with intramedullary cannulated headless screws.J Hand Surg Am.vol.40,pp. 692-7, 2015.

[12] P. Kurzen, C. Fusetti, M. Bonaccio, L. Nagy. Complications after plate fixation of phalangeal fractures. J Trauma.vol.60,pp.8 ,2006.

[13] M.Parag,B.Nimish, P.Vijay and R.Tirthesh. Simple low cost static external fixators for phalangeal fractures of hand IJOS.vol. 4(1),pp.714-720 @ 2018 IJOS,2018,

[14] D. Jafari, A.Ajvadi. A Novel Handmade External Fixator for Phalangeal and Metacarpal Fractures. J. Res. Orthop. Sci.vol. 4 ,pp.8, 2017.

[15] K.Margić . External fixation of closed metacarpal and phalangeal fractures of digits. A prospective study of one hundred consecutive patients. J Hand Surg Br.vol.31(1),pp.3040,2006.

[16] S.Faruqui ,P. Stern,T. Kiefhaber. Percutaneous pinning of fractures in the proximal third of the proximal phalanx: complications and outcomes. JHand Surg Am. vol.37 (7),pp.1342e1348,2012.

[17] J. Singh, K.Jain ,R. Mruthyunjaya, Ravishankar . Outcome of closed proximal phalangeal fractures of the hand. Indian J Orthop.vol.45(5),pp.432e438,2011.

[18] T.Horton,M. Hatton ,T. Davis . A prospective randomized controlled study of fixation of long oblique and spiral shaft fractures of the proximal phalanx: closed reduction and percutaneous Kirschner wiring versus open reduction and lag screw fixation. J Hand Surg Br. 2003;28(1):5-9. 